\title{
LETTER
}

\section{Cardiac output measurements using the bioreactance technique in critically ill patients}

\author{
David Fagnoul, Jean-Louis Vincent and Daniel De Backer*
}

\begin{abstract}
Measurement of cardiac output (CO) using minimally invasive devices has gained popularity. In 11 patients we compared $\mathrm{CO}$ values obtained using the bioreactance technique - a new continuous, totally non-invasive CO monitor - with those obtained by semi-continuous thermodilution using a pulmonary artery catheter. We obtained CO measurements at study inclusion and after any relevant change in hemodynamic status (spontaneous or during fluid challenge, inotrope or vasopressor infusions). There was a poor correlation between the two techniques $(r=0.145)$. These data suggest that caution should be applied when using bioreactance devices in critically ill patients.
\end{abstract}

Measurement of cardiac output $(\mathrm{CO})$ requires use of invasive or minimally invasive devices; the use of noninvasive and minimally invasive devices has gained popularity in recent years. The bioreactance technique is a relatively new, continuous, totally non-invasive technique for measuring $\mathrm{CO}$ that is easily implemented. This new technique involves analyzing phase shifts of a delivered oscillating current that occur when the current traverses the thoracic cavity [1], and differs from traditional bioimpedance techniques that rely on analysis of changes in signal amplitude. Most validation studies in critically ill patients have shown good correlation and/or agreement of bioreactance values compared with $\mathrm{CO}$ values obtained using other devices in patients admitted after cardiac surgery [2-4]. However, validation in critically ill patients is lacking.

As part of the internal evaluation of a bioreactance device before its implementation in the unit (evaluation of new non-invasive monitoring systems before introduction in the unit does not require the approval of the ethics

*Correspondence: ddebacke@ulb.ac.be

Department of Intensive Care, Erasme University Hospital, Route de Lennik 808, Université Libre de Bruxelles, B-1070 Brussels, Belgium
Table 1. Patient characteristics

\begin{tabular}{lc}
\hline Characteristic & $\boldsymbol{n}$ \\
\hline Patients & 11 \\
Cardiogenic shock & 3 \\
Septic shock/distributive shock & 4 \\
Acute respiratory distress syndrome & 4 \\
Therapies & $7(8,2$ to 20) \\
Norepinephrine & $4(5,5$ to 8$)$ \\
Dobutamine & 5 \\
Mechanical ventilation & 2 \\
Hemofiltration & 5 \\
\hline
\end{tabular}

Data in parentheses represent maximal dose, range $(\mu \mathrm{g} /$ minute for norepinephrine and $\mu \mathrm{g} / \mathrm{kg}$.min for dobutamine).

committee in our institution), we compared $\mathrm{CO}$ values obtained using the bioreactance technique (NICOM system; Cheetah Medical Inc., Portland, OR, USA) with those measured using semi-continuous cardiac output by thermodilution (CCO) with a pulmonary artery catheter (Vigilance, Edwards LifeSciences, Irvine, CA, USA). In 11 patients the $\mathrm{CO}$ values were compared at study inclusion and each time a relevant change in hemodynamics and/ or in therapeutics (for example, fluid challenge, inotrope or vasopressor infusions) was observed (Table 1).

We recorded bioreactance CO (average of five values over a 5-minute period) just after obtaining the pulmonary artery catheter $\mathrm{CCO}$ (average of five $\mathrm{CCO}$ values over a 5-minute period). We collected 141 pairs of measurements ( 3 to 23 per patient); the duration of monitoring was at least 3 hours but never exceeded 24 hours. The pulmonary artery catheter $\mathrm{CCO}$ values ranged from 3.9 to $11 \mathrm{l} /$ minute. There was poor correlation between the two techniques (correlation coefficient $r=0.145$ ) (Figure 1 ). To limit the time effect, we randomly selected one pair of measurements for each patient - but this did not improve the results $(r=0.13)$. Bland and Altman analysis with correction for multiple measurements showed wide limits of agreement (Figure 2). The time course of $\mathrm{CO}$ was not well tracked either, sometimes with opposite trends between the two devices. We therefore decided to stop the evaluation. 


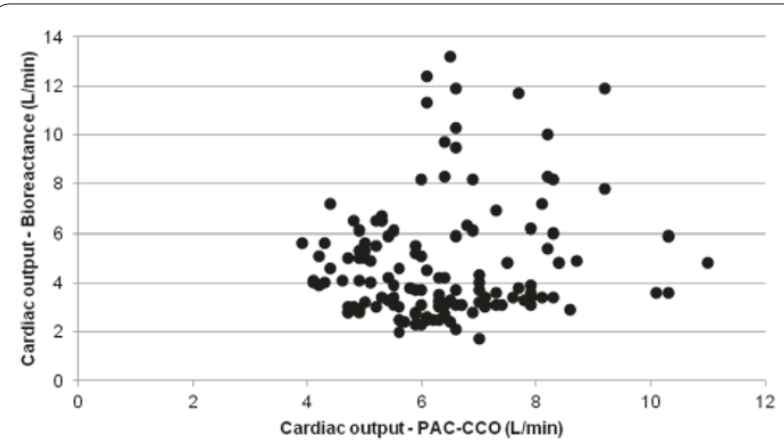

Figure 1. Correlation between pulmonary artery catheter semicontinuous cardiac output by thermodilution and bioreactance cardiac output. A total of 141 measurements in 11 patients, $r=$ 0.1455 . PAC-CCO, pulmonary artery catheter semi-continuous cardiac output by thermodilution.

The bioreactance technique is dependent on diffusion of electrical current, so interstitial edema may interfere with measurements; we believe this is the most probable explanation for the poor correlation. Whatever the reason, these data suggest that caution should be applied when using bioreactance devices in critically ill patients.

\section{Abbreviations}

$\mathrm{CCO}$, semi-continuous cardiac output by thermodilution; $\mathrm{CO}$, cardiac output.

\section{Competing interests}

The authors declare that they have no competing interests.

Published: 9 November 2012

\section{References}

1. Squara P, Denjean D, Estagnasie P, Brusset A, Dib JC, Dubois C: Noninvasive cardiac output monitoring (NICOM): a clinical validation. Intensive Care Med 2007, 33:1191-1194.

2. Marque S, Cariou A, Chiche JD, Squara P: Comparison between

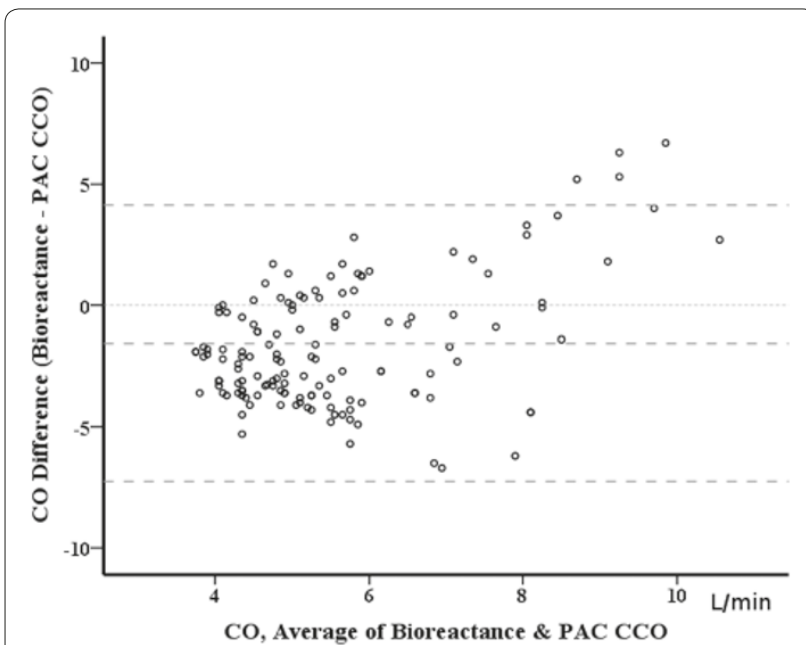

Figure 2. Pulmonary artery catheter semi-continuous cardiac output by thermodilution and bioreactance cardiac output: bias and agreement. A total of 141 pairs of measurements in 11 patients. Bias $-1.6 \mathrm{~L} / \mathrm{min}$ and limits of agreement $5.7 \mathrm{~L} / \mathrm{min}$. CO, cardiac output; PAC-CCO, pulmonary artery catheter semi-continuous cardiac output by thermodilution.

Flotrac-Vigileo and bioreactance, a totally noninvasive method for cardiac output monitoring. Crit Care 2009, 13:R73.

3. Raval NY, Squara P, Cleman M, Yalamanchili K, Winklmaier M, Burkhoff D: Multicenter evaluation of noninvasive cardiac output measurement by bioreactance technique. J Clin Monit Comput 2008, 22:113-119.

4. Benomar B, Ouattara A, Estagnasie P, Brusset A, Squara P: Fluid responsiveness predicted by noninvasive bioreactance-based passive leg raise test. Intensive Care Med 2010, 36:1875-1881.

doi:10.1186/cc11481

Cite this article as: Fagnoul D, et al.: Cardiac output measurements using the bioreactance technique in critically ill patients. Critical Care 2012, 16:460. 